\title{
Bare spot: a normal variant on shoulder MR arthrography
}

\author{
Hee Kyung Kim
}

Received: 26 January 2009 /Revised: 19 February 2009 / Accepted: 6 March 2009 /Published online: 21 April 2009

(C) Springer-Verlag 2009

A 14-year-old boy with shoulder pain after a football injury underwent shoulder MR arthrography. The study revealed a focal area of cartilage thinning (Fig. 1 arrow) at the central area of the glenoid, simulating a cartilage defect. The sagittal image showed a focal contrast-filled area at the central portion of the glenoid (Fig. 2 arrow). This cartilage thinning at the central area of the glenoid represents a bare spot. No abnormality was identified to account for the child's pain.

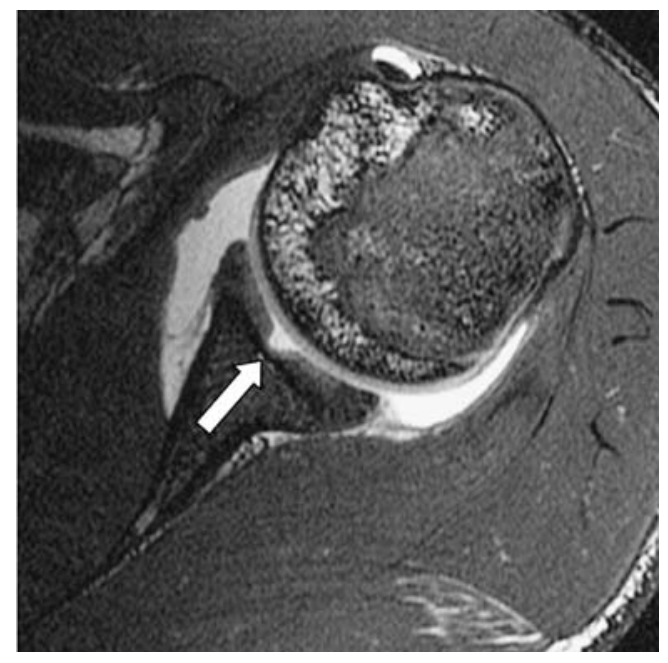

Fig. 1 Axial FIESTA image

H. K. Kim $(\bowtie)$

Department of Radiology,

Cincinnati Children's Hospital Medical Center,

3333 Burnet Ave., MLC5031,

Cincinnati, OH 45229-3039, USA

e-mail: Hee.Kim@cchmc.org

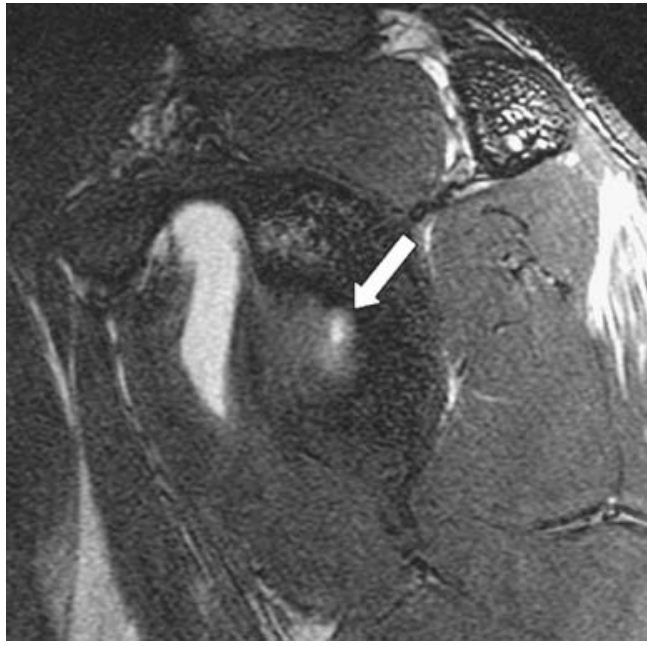

Fig. 2 Sagittal FIESTA image

Normally the articular cartilage of the glenoid has the thinnest portion at its center and is called the "bare spot." It is located at the junction of the middle and lower thirds of the glenoid cavity and used as the arthroscopic landmark. The incidence found in a cadaveric study was $88 \%$ [1]. This typical location of the bare spot can differentiate this normal variant from a cartilage defect [2].

\section{References}

1. Resnick D, Kang HS (2007) Shoulder. In: Resnick D, Kang HS (eds) Internal derangements of joints, 2nd edn. Saunders, Philadelphia, pp 713-1122

2. McCarty LP 3rd, Cole BJ (2005) Nonarthroplasty treatment of glenohumeral cartilage lesions. Arthroscopy 21:1131-1142 